\title{
IMPURITY-DEFECT COMPLEXES IN HYDROGENATED AMORPHOUS SILICON*
}

\author{
L. H. Yang \\ Argonne National Laboratory \\ Materials Science Division \\ Argonne, IL 60439 \\ C. Y. Fong \\ Department of Physics \\ University of California-Davis \\ Davis, CA 95616 \\ C. S. Nichols \\ Department of Materials Science and Engineering \\ Cornell University \\ Ithaca, NY 14853
}

CONF-901105--82

DE9 I 006765
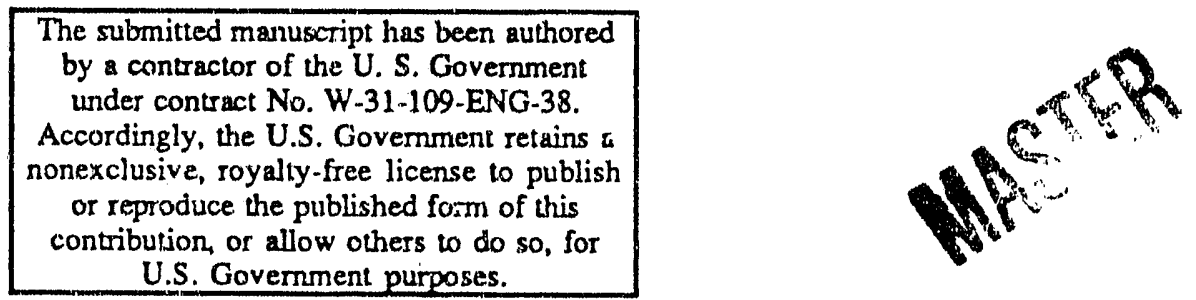

NOVEMBER 1990

\section{DISCLAIMER}

\begin{abstract}
This report was prepared as an account of work sponsored by an agency of the United States Government. Neither the United States Gnvernment nor any agency thereof, nor any of their employees, makes any warranty, express or implied, or assumes any legal liability or responsibility for the accuracy, completeness, or usefuiness of any information, apparatus, product, or process disclosed, or represents that its use would not infringe privately owned rights. Reference herein to any specific commercial product, process, or service by trade name, trademark, manufacturer, or otherwise does not necessarily constitute or imply its endorsement, recommendation, or favoring by the United States Government or any agency thereof. The views and opinions of authors expressed herein do not necessarily state or reflect those of the Uniter. States Government or any agency thereof.
\end{abstract}

Submitted to 1990 Fall Meeting of the Materials Research Society, Bcston, MA, November 26-30, 1990.

*Work Supported by the U.S. Department of Energy, BES-Materials Sciences, under Contract W-31-109-ENG-38.

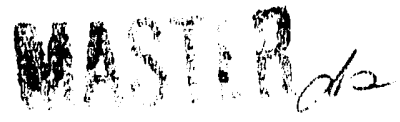

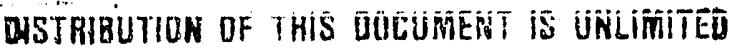




\author{
LIN H. YANG*, C. Y. FONG**, and CAROL S. NICHOLS*** \\ *Materials Science Division, Argonne National Laboratory, Argonne, Il 60439, USA. \\ **Department of Physics, University of California, Davis, CA 95616, USA. \\ ***Department of Materials Science and Engineering, Comell University, Ithaca, NY \\ 14853, USA.
}

\begin{abstract}
The two most outstanding features observed for dopants in hydrogenated amorphous silicon (a-Si:H) - a shift in the Fermi level accompanied by an increase in the defect density and an absence of degenerate doping - have previously been postulated to stem from the formation of substitutional dopant-dangling bond complexes. Using firstprinciples self-consistent pseudopotential calculations in conjunction with a supercell model for the amorphous network and the ability of network relaxation from the first-principles results, we have studied the electronic and structural properties of substitutional fourfoldcoordinated phosphorus and boron at the second neighbor position to a dangling bond defect. We demonstrate that such impurity-defect complexes can account for the general features observed experimentally in doped a-Si: $\mathrm{H}$.
\end{abstract}

\title{
INTRODUCTION
}

The investigation of whether the shallow donor and acceptor states exit in doped hydrogenated amorphous silicon (a- $\mathrm{Si}: \mathrm{H}$ ) has triggered a number of theoretical and experimental studies of the doping mechanism in a-Si:H.[1-8] Among them, Kocka[7] and Kocka et al.[8] have found from constant photocurrent measurements that the neutrai Si dangling bond $\left(D^{0}\right)$ in undoped samples has the same position in the energy gap as the $D^{-}$level in P-doped samples. In B-doped samples, however, they found that the energy of the positively-charged dangling bond $\left(\mathrm{D}^{+}\right)$is $0.4 \mathrm{eV}$ closer to the conduction band edge than is the $\mathrm{D}^{0}$ level. These authors pointed out that their data were consistent with the pairing model between dangling bonds and dopants, but noted that because of the longrange nature of the coulornb interaction, the defect and dopant need not be neares: neighbors. In this paper, we present theoretical results for the electronic structure of a 
fourfold-coordinated $(<4>$ ) substitutional impurity atom paired at the second-neighbor position to a dangling bond defect in an a-Si:H model as schematically shown in Fig. 1. We choose three different configurations in the supercell model which have varying degrees of local bond-angle and bond-length distortions to examine the effects of local geometries on the electronic structures of the defect states. The results are relevant to the understanding of the doping mechanism in a-Si:H.

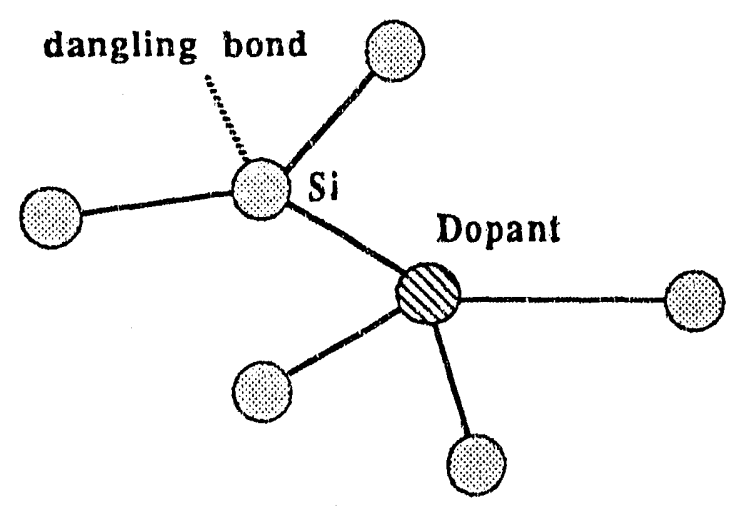

Fig. 1 The configuration of the impurity-defect complex in a-Si:H model.

The dopant can be either $\mathrm{P}$ or $\mathrm{B}$. The dashed line is a dangling bond.

\section{METHODS AND MODELS}

A computer-generated model of a-Si:H with periodic boundary conditions [9] has been used as the basis of our calculations. The model contains $54 \mathrm{Si}$ and $6 \mathrm{H}$ atoms, in which no more than one $\mathrm{H}$ atom is bonded to any $\mathrm{Si}$ atom and all $\mathrm{Si}$ atoms are fourfoldcoordinated. The lattice constant is $10.257 \AA$. The dangling bond was modeled by removing one $\mathrm{H}$ atom from the network. The electronic parts of the calculations are based on the self-corsistent pseudopotential method within the density functional theory, within the local density approximation (LDA). [10] The generalized no,m-conserving pseudopotentials of Hamann [11] and Kleinman-Bylander form [12] for P, B, H, and Si atoms were used. We have employed local optimization techniques for the electronic and the ionic degrees of freedom toward the total energy minimum of the system throughout our calculations. A preconditioned conjugate gradient method [13] is used to obtain solutions of the Schrödinger-like equation, and the Hellmann-Feynman forces are then calculated in conjunction with the conjugate gradient method for the relaxation of the ions. A plane-wave basis set for the potentials and wave functions was utilized with a kinetic energy cut-off of $15 \mathrm{Ry}$, resulting in 7123 plane waves at the sampling $\mathbf{k}=0$ point. 


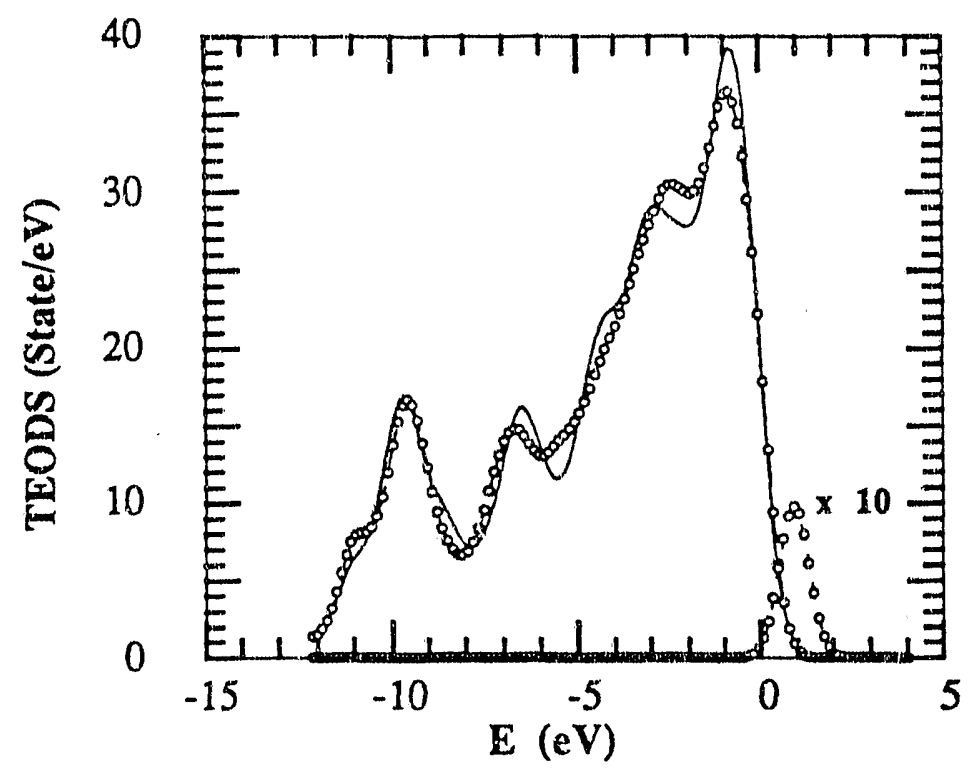

Fig. 2 TEDOS of the a-Si:H model (solid line) and the a-Si:H model containing a dangling bond (open circles). A Gaussian broadening factor of $0.25 \mathrm{eV}$ is used.

\section{RESULTS}

Based on the classical a-Si:H model generated by Guttman and Fong [9], we first relaxed the a-Si:H network according to the Hellmann-Feynman forces that resulted from the self-consistent electronic structure calculations. The total electron density of states (TEDOS) is then calculated to compare with the photoemission experiments performed by von Roedern et al. [14]. Four structures are identified at $-1.3 \mathrm{eV},-3.2 \mathrm{eV},-7.0 \mathrm{eV}$, and $10.0 \mathrm{eV}$ with respect to the top of valence band in our TEDOS data (Fig. 2). They are in reasonably good agreement with the experimental results of $-1.9 \mathrm{eV},-4.5 \mathrm{eV},-7.0 \mathrm{eV}$, and $-10.0 \mathrm{eV}$ [14]. In addition, in our TEDOS data we find a shoulder appears at $-4.7 \mathrm{eV}$ that does not show up in the experimental TEDOS data. The energy gap of the present model is $1.0 \mathrm{eV}$ which is about $0.8 \mathrm{eV}$ smaller than the commonly accepted value of $1.8 \mathrm{eV}$.[15] The properties of the isolated dangling bond have been studied by examining four dangling-bond sites chosen in the model. The average energy of the dangling bond state is $0.55 \pm 0.15 \mathrm{eV}$ above the top of the valence band $\left(E_{\mathrm{v}}+(0.55 \pm 0.15) \mathrm{eV}\right)$, where the 
variation indicates the energy level variation due to the local geometry. This result agrees reasonably well with the experimental data $[16]\left(E_{V}+(0.9 \pm 0.1) \mathrm{eV}\right)$. Especially, the relative position of the $D^{0}$ state, which is sitting in the middle of the energy gap, is consistent with experiment. From the charge density contour plots of the $\mathrm{D}^{0}$ state, we can identify the $\mathrm{D}^{0}$ state as an non-bonding $p$-like state. In Fig. 2, we also show the TEDOS plot of the a-Si:H model containing one dangling bond. In comparing these two plots we can conclude the following features: (i) The dangling-bond states appear in the middle of the energy gap; (ii) The decreasing intensity of the first peak, at $-1.3 \mathrm{eV}$, indicates the merging of the Si $p$-like state into the energy gap; (iii) The changing positions and intensities of the second and the third peaks suggest that they are $\mathrm{H}$-related states.

When the dangling bond is paired with a $<4>\mathrm{P}$ atom at the second neighbor site, the average energy of the resulting $D^{-}$states is $0.05 \mathrm{eV}$ lower than the $\mathrm{D}^{0}$ state, in good agreement with the constant photocurrent results.[7,8] More importantly, however, the average energy of the $\mathrm{P}_{4}^{+}$states moves well up into the conduction bands. As compared to the charge derisity of the $D^{0}$ state, the center of mass of the $D^{-}$charge density is moved toward the $\mathrm{P}$ atom by 0.06 a.u. By examining the charge density the $\mathrm{D}^{-}$state, we find that this state is essentially an $s-p$ hybrid in character and there is a $26 \%$ increase in the charge as compared to the neutral uangling bond state. The results suggest that the interaction between the $\mathrm{D}^{-}$state and the $\mathrm{P}_{4}^{+}$ion causes the charge density of the $\mathrm{D}^{-}$state spreading more than that of the $\mathrm{D}^{0}$ state. As a result the donor electron is trapped by the neutral danglingbond defect site and thus the formation of shallow donor state is not available under the present doping scheme.

To study the $\mathrm{B}$-dangling bond pair, we replaced the $\mathrm{P}$ atom by a $\mathrm{B}$ atom. we find the energy level of the $\mathrm{B}_{4}$ atom merges well into the valence band. Shallow acceptor states do not appear in the dopant-dangling bond complex. The average energy of the $\mathrm{D}^{+}$states moves upward by $0.30 \pm 0.05 \mathrm{eV}$ with respect to that of the $D^{0}$ stares. Kocka et al.[7,8] also found in their constant photocurrent measurements that the $\mathrm{D}^{+}$states shifted upward by $0.4 \mathrm{eV}$ relative to the $\mathrm{D}^{0}$ levels. The qualitative feature that the energy of the $\mathrm{D}^{+}$level is higher than that of the $D^{0}$ state is consistent with experiment. As compared to the $D^{0}$ reference state, the $\mathrm{D}^{+}$state has a more extended charge distribution because of its antibonding character. The center of mass of the $\mathrm{D}^{+}$state is moved away from the $\mathrm{B}$ atom by 0.83 a.u. with respect to the $D^{0}$ state. Thes $s-p$ hybridization character of the posi.ively 
charged defect state $\left(\mathrm{D}^{+}\right)$is manifested clearly by the shift in the charge density toward the dopant atom.

\section{SUMMARY}

In conclusion, the electronic properties of $\mathrm{P}_{4}^{+}-\mathrm{D}^{-}$and $\mathrm{B}_{4}^{-}-\mathrm{D}^{+}$pairs in a-Si:H were calculated by the local optimization techniques for the electrons and the ions and compared with those of the $\mathrm{D}^{0}$ state. For each case, three pairs at different sites (and hence with different local distortions) in a-Si:H were examined. The average energies of $\mathrm{D}^{-}$and $\mathrm{D}^{+}$ with respect to the $D^{0}$ states agree well with experimental measurements, especially the occupied states. The missing of shallow donor and acceptor states suggests that the doping efficiency is low in a-Si:H. Furthermore, the good agreement between theory and experiment on the energy shift of the $D^{-}$state relative to the $D^{0}$ state demonstrates that our rechniques can be applied to the study of the electronic structures of the occupied defect states in an efficient fashion. We summarize the theoretical and the experimental results in Table I.

Table 1. The average energies and participation ratios of the dangling-bond defect states. $E_{D}{ }^{0}$ is the $D^{0}$ state energy level and $E_{v}$ is the energy of the top of the valence band. The participation ratio, which gives the guideline whether the electronic states is localized or extendeci, is calculated according to $\frac{1}{\Omega \int\left|\phi_{i}(r)\right|^{4} \mathrm{~d} \mathbf{r}}$, where $\phi_{i}(r)$ is the $i$-th electron wave function and $\Omega$ is the volume of the supercell. The unit cell volume is $1079.10 \AA^{3}$.

\begin{tabular}{|c|c|c|c|}
\hline Species & Energy level & Experiment & Participation ratio \\
\hline$D^{0}$ & $E_{\mathrm{v}}+(0.55 \pm 0.15) \mathrm{eV}$ & $\mathrm{E}_{\mathrm{V}}+(0.9 \pm 0.1) \mathrm{eV}[16]$ & $8.85 \%$ \\
\hline $\mathrm{D}^{-}$ & $\mathrm{E}_{\mathrm{D}}{ }^{0}-(0.05 \pm 0.10) \mathrm{eV}$ & $\mathrm{E}_{\mathrm{D}}^{0}[7,8]$ & $9.20 \%$ \\
\hline $\mathrm{D}^{+}$ & $\mathrm{E}_{\mathrm{D}}{ }^{0}+(0.30 \pm 0.05) \mathrm{eV}$ & $\mathrm{E}_{\mathrm{D}}{ }^{0}+0.4 \mathrm{eV}[7,8]$ & $15.30 \%$ \\
\hline
\end{tabular}




\section{ACKNOWLEDGMENTS}

One of us (L.H.Y.) was supported by the U.S. DOE, BES-Materials Science under contract No. W-31-109-ENG-38 and computing resources on DOE ER/BES Crays. Work at Davis was supported in part by the San Diego Supercomputer Center.

\section{REFERENCE}

1. R. A. Street, Phys. Rev. Lett. 49, 1187 (1982); J. Non-Cryst. Sol. Z7\&.78, 1 (1985).

2. J. Robertson, Phys. Rev. B28, 4647 (1983).

3. I. Chen and F. Jansen, Phys. Rev. B29, 3759 (1984).

4. J. Robertson, Phys. Rev. B31, 3817 (1985).

5. R. A. Street, D. K. Biegelsen, and J. C. Knights, Phys. Rev. B24, 265 (1981).

6. H. Dersch, J. Stuke, and J. Biechler, Phys. Status Solidi B105, 265 (1981).

7. J. Kocka, J. Non-Cryst. Sol. 20, 91 (1987).

8. J. Kocka, M. Vanecek, and F. Schauer, J. Non-Cryst. Sol. 27\&98, 715 (1987).

9. L. Guttman and C. Y. Fong, Phys. Rev. B26, 6756 (1982).

10. P. Hohenberg and W. Kohn, Phys. Rev. 136, B864 (1964); W. Koin and L. J. Sham, Phys. Rev. 140, A1133 (1965).

11. D. R. Hamann, Phys. Rev. B40, 2980 (1989).

12. L. Kleinman and D. M. Bylander, Phys. Rev. Lett. 48, 1425 (1982).

13. M. P. Teter, M. C. Payne, and D. C. Allan, Phys. Rev. B40, 12255 (1989).

14. B. von Roedern, L. Ley, M. Cardona, and F. W. Smith, Phil. Mag. B40, 433 (1979).

15. D. Adler, in Physical Properties of Amorphous Materials, edited by D. Adler, B. B. Schwartz, and M. C. Steele (Plenum, New York, 1985), pg. 5 .

16. A. V. Gelatos, K. K. Mahvadi, J. D. Cohen, and J. P. Harbison, Appl. Phys. Lett. 53, 403 (1988). 

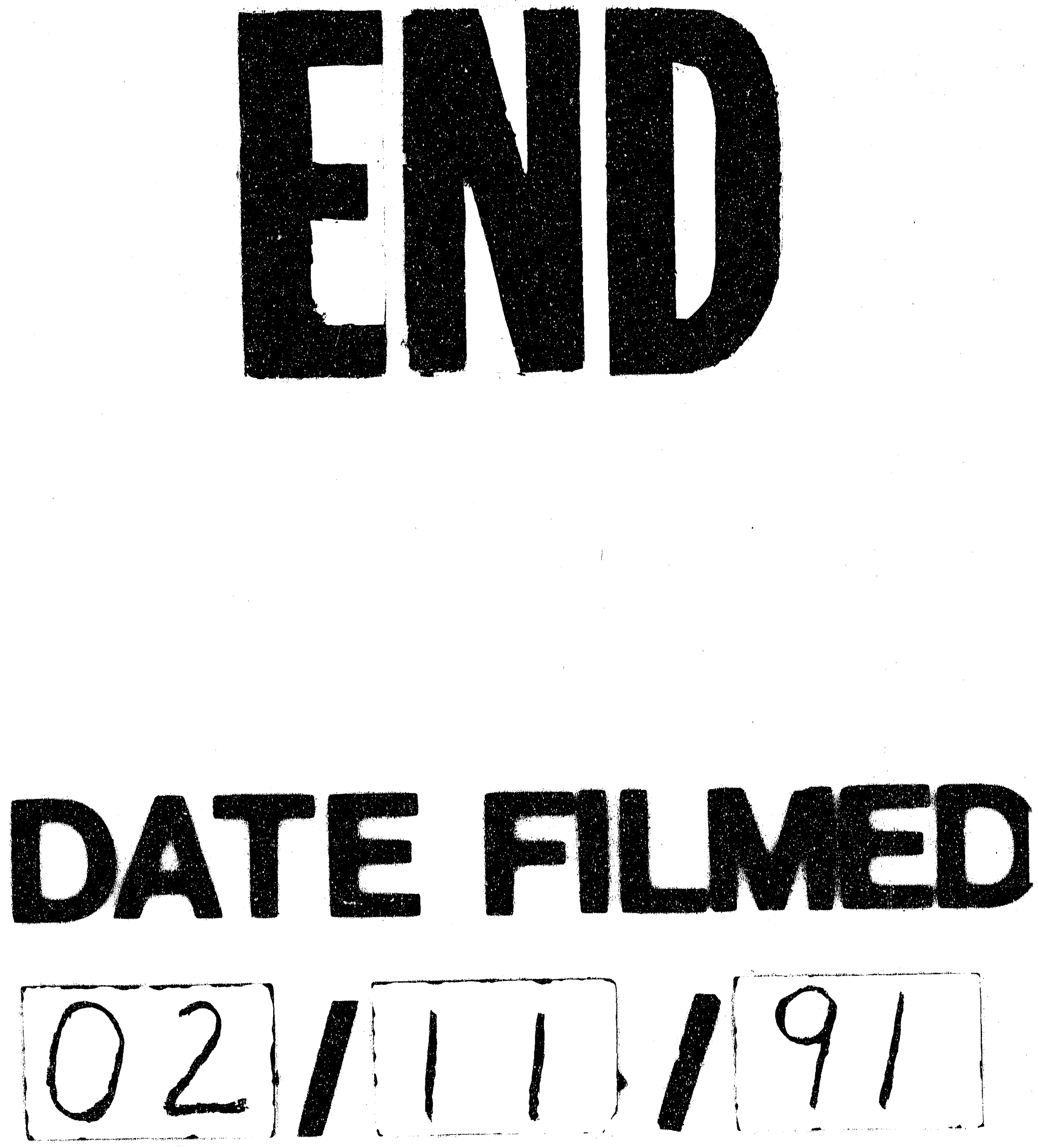
